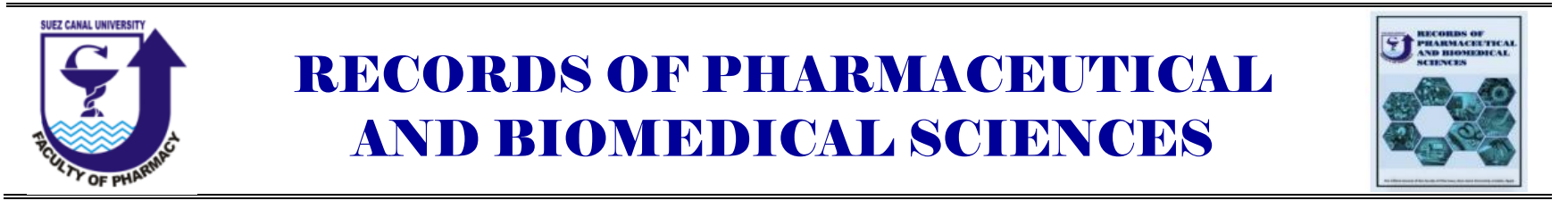

\title{
Self - Emulsifying Drug Delivery System: A Novel Approach for Oral Delivery of Poorly Water Soluble Drugs
}

\author{
Ahmed R.Gardouh ${ }^{a, b}$, Ahmed M.Nasef $^{c^{*}}$,Yasser Mostafa ${ }^{d}$, Shadeed Gad ${ }^{a}$ \\ ${ }^{a}$ Department of Pharmaceutics and Industrial Pharmacy, Faculty of Pharmacy, Suez Canal University, \\ Ismailia, 41522, Egypt; ${ }^{b}$ Department of Pharmacy, Faculty of pharmacy, Jadara University, 21110 Irbid, \\ Jordan; ${ }^{c}$ Production department, Medical Union Pharmaceutical (MUP) Co., Ismailia, 41617, Egypt; \\ ${ }^{d}$ Department of Pharmacology and Toxicology, Faculty of Pharmacy, Suez Canal University, Ismailia, \\ 41522, Egypt.
}

Received on: 09. 12. 2020

Revised on: 20. 01. 2021

Accepted on: 25. 01. 2021

*Correspondence Author:

Tel. : 01008048620

E-mail address:

a_nasef888@yahoo.com

\begin{abstract}
Self nano emulsifying drug delivery systems (SNEDDs) gained much attention in the last decades since, such systems considered one of the most favorable and efficient approaches to enhance solubility, increase drug absorption and hence, enhance its oral bioavailability of poorly water soluble (lipophilic) drug moieties. Owing to their unique properties and advantages over the other conventional dosage forms, many literatures investigate the change physicochemical properties, pharmacokinetic and pharmacodynamics of self emulsifying formulation compared to both pure drug and its conventional dosage form and always reported significant improvement using SNEDDs. This review discus the composition, advantages and mechanism of action of liquid SNEDDs and some techniques used for solidification of liquid formulations to overcome its drawbacks.
\end{abstract}

Keywords: Oral bioavailability, Self nanoemulsifying drug delivery systems, poorly water soluble drugs.

\section{Introduction}

Despite of being the most favorable administration route, it is restricted for drugs with sufficient permeability through gastric mucosa and water solubility. Regarding FDA reports from 1995 to 2002 , only $9 \%$ of the new drug molecules showed high solubility and permeability as such molecules belong to belonged to BCS class-I category. More than $40 \%$ of new drugs entities showed poor oral bioavailability, absence of dose proportionality and high subject variability(Agarwal, Siddiqui et al. 2009).
One of the most difficult challenges in pharmaceutical industry is the formulation of poorly water soluble compounds and enhancing its oral bioavailability. It was reported that one of the most efficient strategy to enhance oral bioavailability and hence, increase the clinical efficiency of lipophilic drug moieties is through incorporation of active lipophilic molecules in suitable lipid vehicle such as liposomes, oils and self-emulsifying formulations(Gershanik and Benita 2000).

Self-nanoemulsifying drug delivery systems 
(SNEDDs)are isotropic mixtures of synthetic and /or natural oils, surfactants (liquid / solid), hydrophilic solvent(s) and cosolvent/surfactant(Myers and Stella 1992, Shah, Carvajal et al. 1994).

The drug molecules incorporated in SNEDDs must be partitioned within the fine o/w emulsion droplets formed upon contact with aqueous fluids in (GIT) to avoid poor in-vivo performance resulted from precipitation of drug and this can be avoided via using high concentration of surfactants(Wei, Ye et al. 2012).

Self-nanoemulsifying systems have shown lots of unique properties and advantages over the other conventional delivery systems such as(Patel, Patel et al. 2010, Kaur 2013):
- Upon contact with aqueous fluids, very fine $\mathrm{O} / \mathrm{W}$ emulsion droplets are formed which pass rapidly facilitating drug distribution throughout stomach and improve drug absorption from GIT, thereby diminish irritation caused by prolonged contact between drug substances and GIT wall.

- SNEDDs are physically stable compared to conventional sensitive emulsions that are metastable dispersed forms.

- Provide a large interfacial area for drug partitioning between phases (oily and aqueous phase) compared to solutions of oily nature.

- Selective targeting of drug toward its absorption site in GIT.

- Minimize variability such as food effects.

- High drug loading efficiency and ability to enhance oral bioavailability with reduced dose.

Table 1: Marketed preparations of self emulsifying drug delivery systems (SEDDs (Wadhwa, Nair et al. 2012)

\begin{tabular}{|l|l|l|l|}
\hline $\begin{array}{c}\text { Trade Name } \\
\text { (Company) }\end{array}$ & Drug Molecule & \multicolumn{1}{|c|}{ Type of formulation } & \multicolumn{1}{c|}{ Excipients } \\
\hline $\begin{array}{l}\text { Sandimmune } \\
\text { (Novartis) }\end{array}$ & Cyclosporin A & $\begin{array}{l}\text { Oral solution } \\
(100 \mathrm{mg} / \mathrm{ml})\end{array}$ & $\begin{array}{l}\text { Olive oil, polyoxyethylated oleic glycerides } \\
\text { (Labrafil M 1944 CS) }\end{array}$ \\
\hline $\begin{array}{l}\text { Gengraf } \AA \\
(\text { Abbott })\end{array}$ & Cyclosporin A & $\begin{array}{l}\text { Soft gelatin capsule } \\
(25,100 \mathrm{mg})\end{array}$ & $\begin{array}{l}\text { Polyoxyl 35 castor oil (Cremophor EL), } \\
\text { polysorbate } 80\end{array}$ \\
\hline $\begin{array}{l}\text { Kaletra } \\
(\text { Abbott })\end{array}$ & $\begin{array}{l}\text { Lopinavir \& } \\
\text { Ritonavir }\end{array}$ & $\begin{array}{l}\text { Oral solution lopinavir } \\
(80 \mathrm{mg} / \mathrm{ml}) \& \\
\text { ritonavir }(20 \mathrm{mg} / \mathrm{ml})\end{array}$ & $\begin{array}{l}\text { Polyoxyl hydrogenated castor oil } \\
\text { (Cremophor RH 40), peppermint oil }\end{array}$ \\
\hline $\begin{array}{l}\text { Norvir }{ }^{\circledR} \\
(\text { Abbott) }\end{array}$ & Ritonavir & $\begin{array}{l}\text { Soft gelatin capsule }(100 \\
\mathrm{mg})\end{array}$ & $\begin{array}{l}\text { Oleic acid, polyoxyl 35 castor oil (Cremophor } \\
\text { EL) }\end{array}$ \\
\hline
\end{tabular}

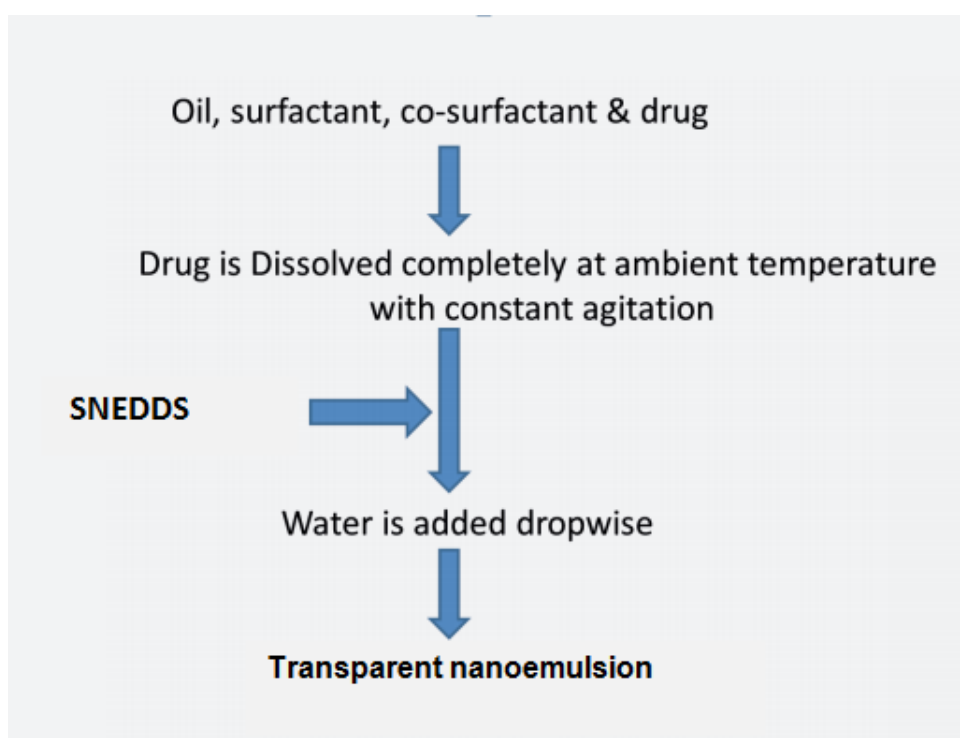

Figure 1: Flow chart for SNEDDs preparation 
Composition of self-emulsifying drug delivery systems:(Neslihan Gursoy and Benita 2004)

The self-emulsifying process is based on:

- Concentration of surfactant.

- Nature of surfactant-oil phase combination

- The temperature at which selfemulsification process occurs

\section{1- Drug/active pharmaceutical ingredient} According to the Biopharmaceutical classification system (BCS), there are four classes of drugs based on solubility (ability of a solute to dissolve in a solvent) and permeability (contact between a solute and solvent to form a solution)(Khedekar and Mittal 2013).SEDDs usually used to enhance absorption related to the poor permeability and/ or solubility of the Biopharmaceutical Classification System (BCS) class II to IV drugs. However, such systems can be used for the four classes(Singh, Bandopadhyay et al. 2009).

\section{2- Oils}

It is the most critical component as it increase the absorption of included drug from GIT through facilitating self-emulsification process and increasing lipophilic drug fraction transported through the intestinal lymphatic system (Khoo 1998).

Both long and medium chain triglyceride (LCT and MCT) oils with variable saturation degrees have been used for manufacturing of self-emulsifying systems.

Semisynthetic MCT, which are amphiphilic compounds with surfactant properties are widely replacing the common medium-chain triglyceride oils(Constantinides 1995).

\section{3- Surfactant}

Since the self-emulsification property of SNEDDs is the crucial process to form the fine emulsion droplets, a surfactant is needed to adopt such property, dissolve / solubilize include amounts of lipophilic drug, prevent the risk of drug precipitation in GIT and hence, improve dissolution rate (Constantinides 1995).

The selection of surfactant is based on HLB value where, surfactants have a high HLB value help in the rapid spreading of formula in aqueous media and the instant formation of $\mathrm{o} / \mathrm{w}$ droplets. Nonionic surfactants are most preferred due to more favorable safety margins, superior stability over a wider range of $\mathrm{pH}$ and reversible changes in intestinal mucosal permeability(Gupta, Kesarla et al. 2013).

\section{4- Co-surfactant}

Co-surfactant of HLB value (10-14) is usually used in SEDDs as they allow spontaneous formation of very fine $\mathrm{O} / \mathrm{W}$ emulsion droplets through reducing oil-water interface.

\section{Mechanism of action}

In case of conventional emulsions, the free energy is essential for the newly formed surface between the two immiscible phases (aqueous and oily phase). Because of high energy, the emulsion may become unstable and the phases separate in order to reduce the interfacial area so, an emulsifying agent is usually added to reduce the interfacial energy, prevent coalescence and stabilize the emulsion(Reiss 1975, Constantinides 1995).

In case of SNEDDs, the fine emulsion droplets formation occurs immediately because the very low free energy of the system resulted from the existence of flexible interface. Upon mild agitation, oil and surfactant/ co-surfactant mixture is mixed with aqueous phase and the aqueous phase penetrate the newly formed interface between the two phases and solubilize the oil (Akula, Gurram et al. 2014).

Development of solid self-nanoemulsifying drug delivery system (S-SNEDDs)

Most of SNEDDs are in liquid state as major ingredients (oil, surfactants and co-surfactants) used in SNEDDs are usually in liquid state at room temperature. The liquid nature of SNEDDs limits the wide application of such technology for enhancing the oral bioavailability of various drugs because of many disadvantages including lack of stability, large dose volume because of relatively low drug loading capacity, irreversible precipitation of drug and migration of volatile ingredients into shell of gelatin capsule (Memvanga and Préat 2012, Tarate, Chavan et al. 2014).

Solidification of Liquid SNEDDS is an efficient strategy to overcome the restrictions of liquid SNEDDS and combine the benefits of SNEDDs (enhancement of both solubility and bioavailability) with the advantages of solid dosage forms (ease of handling and portability, higher stability and reproducibility, compact dosage form and better patient compliance). Regarding the advantages of solid dosage forms, S-SNEDDs have been widely considered in the recent few years, as they considered more efficient alternatives to conventional liquid SNEDDs.

\section{Solidification techniques}

Capsule filled with semi solid / liquid formula

The advantages of simplicity, high drug loading potential make this method is the most common technique for developing solid self-emulsifying formulations where, either semi-solid and / or 


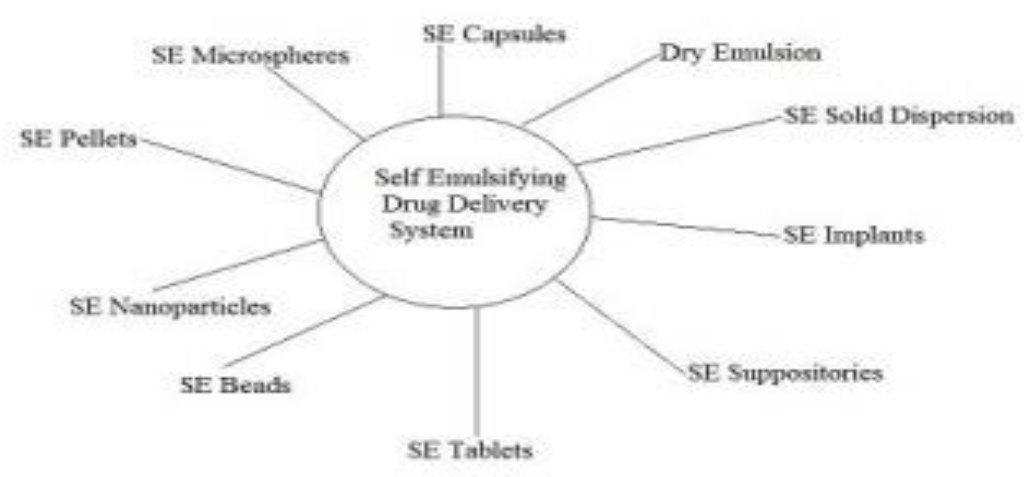

Figure 2: Various types of solid SEDDs

liquid self-emulsifying formula is filled into the soft or hard gelatin capsules for oral administration. The factor that governs the filling into capsule is the compatibility of formula filled with the capsule shell. An alternative method should be used if the formulation contains volatile oils as such components tend to leak through the capsule shell.

For liquid self emulsification formulations, the Liquid- Oros technology is applied, this technology used osmotic properties where the layer swells upon contact with aqueous media and is pumped into the hard or soft gelatin capsules(Shukla 2016).

For the semi-solid self emulsifying formulations, the semisolids are heated to a temperature twenty degrees above their melting point., filled into capsule body which then capped and left to cool at room temperature (Pouton and Porter 2008, Tarate, Chavan et al. 2014).

\section{Spray drying}

This method includes the addition of the liquid SNEDDs to the solution of a suitable solid carrier which may be hydrophilic (e.g. dextran) or hydrophobic (e.g. colloidal silica)(Oh, Kang et al. 2011). The obtained solution is then atomized to very fine droplets in the drying chamber, where the volatile solvent (water and /or organic solvent) evaporates, forming dry particles under controlled temperature and airflow conditions (Nicolaos, Crauste-Manciet et al. 2003, Balakrishnan, Lee et al. 2009). The produced dry emulsion powder can be used to prepare self emulsifying tablets or capsules (Kumar, Sharma et al. 2010) solve the problems of drug stability, and usage of toxic organic solvents (Jannin, Musakhanian et al. 2008).

\section{Adsorption on solid carrier}

It is a simple technique in which the liquid SNEDDs are mixed with suitable carrier to produce solid SNEDDs. The selected solid carrier must adsorb large volume of liquid self-emulsifying formulation, and the resulting freely flowing powder can be filled directly into capsules or, alternatively, be compressed into tablets after mixing with suitable excipients.

The produced powder provide good content uniformity but, with low drug loading capacity (Jannin, Musakhanian et al. 2008).

\section{Lyophilization Technique}

Lyophilization technology (also known as freeze drying) involves mass and heat transfer from and to the formulation. Such technique is suitable to be used for drugs sensitive to oxygen, air and temperature. The main drawback of this technique is its high operation cost.

\section{Drawbacks of the solidification technologies}

There are various problems related to the application of solidification technologies such as (Gupta, Kesarla et al. 2013):

1) Amount of solidifying excipients may affect the release of the drug.

2) Nature of the excipients used may affect the drug absorption.

3) Probability of irreversible phase separation on reconstitution.

4) Decomposition of sensitive drug moieties during solidification process.

5) Relatively low drug loading capacity.

Tactics to overcome the problems caused by solidification technologies

1. The gelled SNEDDs has been developed to minimize the amount of solidifying agent required to convert the Liquid SNEDDs into solid dosage form. Owing to its advantages including decreasing the amount of solidifying agent and control the drug release pattern, colloidal silicon dioxide (Aerosil 200 ${ }^{\circledR}$ ) is the most common used gelling agent.(Patil, Joshi et al. 2004).

2. Solid self-emulsifying dispersions: this technology include the dispersion of drugs into 
solid self-emulsifying excipients such as tocopheryl polyethylene glycol 1000 succinate (TPGS), Gelucire150/02 and Gelucire1 44/14 since such excipients significantly improve the absorption of poorly water soluble lipophilic drugs (Serajuddin, Mufson et al. 1988, Betageri and Makarla 1995, M. 1999).

Self emulsifying drug delivery systems for oral drug delivery of poorly soluble drugs

Upon contact with fluids in gastrointestinal tract (GIT), these systems emulsify rapidly and spontaneously to form fine $\mathrm{O} / \mathrm{W}$ emulsions under mild agitation / digestive motility (Iosio, Voinovich et al. 2008, Kaur 2013) generating a large surface area that provide optimum conditions to enhance absorption / bioavailability of poorly soluble compounds and hence, the clinical efficiency $\left(\mathrm{Wu}_{\text {, }}\right.$ Wang et al. 2006, Patil, Patil et al. 2007, Mandawgade, Sharma et al. 2008, Yi, Wan et al. 2008, Cui, Yu et al. 2009).

Many researchers have been reported on SNEDDs (either in liquid or solid form) to improve oral bioavailability of poorly water soluble drugs. For instance:

- R.Gardouh, A., et al.2020 who prepared liquid self nanoemulsifying drug delivery system of Atorvastatin and Ezetimibe combination and investigate its physicochemical properties, pharmacokinetics and pharmacodynamic characteristics and reported a significant enhancement of dissolution rate, drug absorption and lipid lowering activity of included drugs compared to pure drug suspension (R.Gardouh, Nasef et al. 2020).

- Selvam, R.P.,et al.2013 who prepared self nanoemulsifying drug delivery system of poorly water soluble drug (Efavirenz) and reported significant improvement of solubility and dissolution rate(Selvam, Kulkarni et al. 2013).

- Cho, H.-Y., et al., 2016 who prepared solid self nanoemulsifying drug delivery systems of Paclitaxel and reported that such systems are efficient for bioavailability enhancement and targeting drug delivery to lymphatic system (Cho, Kang et al. 2016)

\section{References:}

Agarwal, V., A. Siddiqui, H. Ali and S. Nazzal (2009). "Dissolution and powder flow characterization of solid self-emulsified drug delivery system (SEDDS)." Int J Pharm 366(1-2): 44-52.

Akula, S., A. K. Gurram and S. R. Devireddy
(2014). "Self-Microemulsifying Drug Delivery Systems: An Attractive Strategy for Enhanced Therapeutic Profile." International Scholarly Research Notices 2014: 964051.

Balakrishnan, P., B.-J. Lee, D. H. Oh, J. O. Kim, M. J. Hong, J.-P. Jee, J. A. Kim, B. K. Yoo, J. S. Woo, C. S. Yong and H.-G. Choi (2009). "Enhanced oral bioavailability of dexibuprofen by a novel solid Self-emulsifying drug delivery system (SEDDS)." European Journal of Pharmaceutics and Biopharmaceutics 72(3): 539-545.

Betageri, G. V. and K. R. Makarla (1995). "Enhancement of dissolution of glyburide by solid dispersion and lyophilization techniques." International Journal of Pharmaceutics 126(1): 155160.

Cho, H.-Y., J.-H. Kang, L. Ngo, P. Tran and Y.-B. Lee (2016). "Preparation and Evaluation of SolidSelf-Emulsifying Drug Delivery System Containing Paclitaxel for Lymphatic Delivery." Journal of Nanomaterials 2016: 3642418.

Constantinides, P. P. (1995). "Lipid microemulsions for improving drug dissolution and oral absorption: physical and biopharmaceutical aspects." Pharm Res 12(11): 1561-1572.

Cui, J., B. Yu, Y. Zhao, W. Zhu, H. Li, H. Lou and G. Zhai (2009). "Enhancement of oral absorption of curcumin by self-microemulsifying drug delivery systems." Int J Pharm 371(1-2): 148-155.

Gershanik, T. and S. Benita (2000). "Selfdispersing lipid formulations for improving oral absorption of lipophilic drugs." Eur J Pharm Biopharm 50(1): 179-188.

Gupta, S., R. Kesarla and A. Omri (2013). "Formulation strategies to improve the bioavailability of poorly absorbed drugs with special emphasis on self-emulsifying systems." ISRN Pharm 2013: 848043.

Gupta, S., R. Kesarla and A. Omri (2013). "Formulation strategies to improve the bioavailability of poorly absorbed drugs with special emphasis on self-emulsifying systems." ISRN pharmaceutics 2013: 848043-848043.

Iosio, T., D. Voinovich, M. Grassi, J. F. Pinto, B. Perissutti, M. Zacchigna, U. Quintavalle and F. 
Serdoz (2008). "Bi-layered self-emulsifying pellets prepared by co-extrusion and spheronization: influence of formulation variables and preliminary study on the in vivo absorption." Eur J Pharm Biopharm 69(2): 686-697.

Jannin, V., J. Musakhanian and D. Marchaud (2008). "Approaches for the development of solid and semi-solid lipid-based formulations." Adv Drug Deliv Rev 60(6): 734-746.

Kaur, M. (2013). "Self emulsified drug delivery system for the enhancement of oral bioavailability of poorly water soluble drugs." International journal of advances in pharmacy, biology and chemistry.

Khedekar, K. and S. Mittal (2013). Self emulsifying drug delivery system: A review.

Khoo, S. M., et al. (1998). "Formulation design and bioavailability assessment of lipidicsilfemulsifying formulation of halofanitrine." Int J Pharm. 167: 155-164.

Kumar, A., S. Sharma and R. Kamble (2010). Self emulsifying drug delivery system (SEDDS): Future aspects.

M., S. A. T. (1999). "Solid dispersion of poorly water-soluble drugs: Early promises, subsequent problems, and recent breakthroughs." Journal of Pharmaceutical Sciences 88(10): 1058-1066.

Mandawgade, S. D., S. Sharma, S. Pathak and V. B. Patravale (2008). "Development of SMEDDS using natural lipophile: application to beta-Artemether delivery." Int J Pharm 362(1-2): 179-183.

Memvanga, P. B. and V. Préat (2012). "Formulation design and in vivo antimalarial evaluation of lipidbased drug delivery systems for oral delivery of $\beta$ arteether." European journal of pharmaceutics and biopharmaceutics : official journal of Arbeitsgemeinschaft fur Pharmazeutische Verfahrenstechnik e.V 82(1): 112-119.

Myers, R. A. and V. J. Stella (1992). "Systemic bioavailability of penclomedine (NSC-338720) from oil-in-water emulsions administered intraduodenally to rats." International Journal of Pharmaceutics 78(1): 217-226.

Neslihan Gursoy, R. and S. Benita (2004). "Selfemulsifying drug delivery systems (SEDDS) for improved oral delivery of lipophilic drugs."
Biomedicine \& Pharmacotherapy 58(3): 173-182. Nicolaos, G., S. Crauste-Manciet, R. Farinotti and D. Brossard (2003). "Improvement of cefpodoxime proxetil oral absorption in rats by an oil-in-water submicron emulsion." International Journal of Pharmaceutics 263(1): 165-171.

Oh, D. H., J. H. Kang, D. W. Kim, B. J. Lee, J. O. Kim, C. S. Yong and H. G. Choi (2011). "Comparison of solid self-microemulsifying drug delivery system (solid SMEDDS) prepared with hydrophilic and hydrophobic solid carrier." Int J Pharm 420(2): 412-418.

Patel, S. N., D. M. Patel, C. N. Patel, T. D. Patel, P. H. Prajapati and B. N. Parikh (2010). "Self emulsifying drug delivery system." Journal of Global Pharma Technology 2: 29-37.

Patil, P., P. Joshi and A. Paradkar (2004). "Effect of formulation variables on preparation and evaluation of gelled self-emulsifying drug delivery system (SEDDS) of ketoprofen." AAPS PharmSciTech 5(3): 43-50.

Patil, P., V. Patil and A. Paradkar (2007). "Formulation of a self-emulsifying system for oral delivery of simvastatin: in vitro and in vivo evaluation." Acta Pharm 57(1): 111-122.

Pouton, C. W. and C. J. Porter (2008). "Formulation of lipid-based delivery systems for oral administration: materials, methods and strategies." Adv Drug Deliv Rev 60(6): 625-637.

R.Gardouh, A., A. Nasef, Y. Mostafa and S. Gad (2020). "Design and evaluation of combined atorvastatin and ezetimibe optimized self-nano emulsifying drug delivery system." Journal of Drug Delivery Science and Technology 60: 102093.

Reiss, H. (1975). "Entropy-induced dispersion of bulk liquids." Journal of Colloid and Interface Science 53(1): 61-70.

Serajuddin, A. T. M., D. Mufson, D. F. Bernstein, P.-C. Sheen and M. A. Augustine (1988). "Effect of Vehicle Amphiphilicity on the Dissolution and Bioavailability of a Poorly Water-Soluble Drug from Solid Dispersions." Journal of Pharmaceutical Sciences 77(5): 414-417.

Shah, N. H., M. T. Carvajal, C. I. Patel, M. H. Infeld and A. W. Malick (1994). "Self-emulsifying 
drug delivery systems (SEDDS) with polyglycolyzed glycerides for improving in vitro dissolution and oral absorption of lipophilic drugs." International Journal of Pharmaceutics 106(1): 1523.

Singh, B., S. Bandopadhyay, R. Kapil, R. Singh and O. Katare (2009). "Self-emulsifying drug delivery systems (SEDDS): formulation development, characterization, and applications." Crit Rev Ther Drug Carrier Syst 26(5): 427-521.

Tarate, B., R. Chavan and A. K. Bansal (2014). "Oral solid self-emulsifying formulations: a patent review." Recent Pat Drug Deliv Formul 8(2): 126143.

Wadhwa, J., A. Nair and R. Kumria (2012). "Emulsion forming drug delivery system for lipophilic drugs." Acta poloniae pharmaceutica 69: 179-191.

Wei, Y., X. Ye, X. Shang, X. Peng, Q. Bao, M. Liu, M. Guo and F. Li (2012). "Enhanced oral bioavailability of silybin by a supersaturatable selfemulsifying drug delivery system (S-SEDDS)." Colloids and Surfaces A: Physicochemical and Engineering Aspects 396: 22-28.

Wu, W., Y. Wang and L. Que (2006). "Enhanced bioavailability of silymarin by selfmicroemulsifying drug delivery system." Eur $\mathbf{J}$ Pharm Biopharm 63(3): 288-294.

Yi, T., J. Wan, H. Xu and X. Yang (2008). "Controlled poorly soluble drug release from solid self-microemulsifying formulations with high viscosity hydroxypropylmethylcellulose." Eur J Pharm Sci 34(4-5): 274-280. 